Journal of the Operations Research

Society of Japan

Vol. 44, No. 3, September 2001

\title{
DETERMINATION OF OPTIMAL REPAIR-COST LIMIT ON THE LORENZ CURVE
}

\author{
Tadashi Dohi \\ Hiroshima University
}

\author{
Naoto Kaio \\ Hiroshima Shudo University
}

\author{
Shunji Osaki \\ Nanzan University
}

(Received April 15, 1999; Revised August 14, 2000)

\begin{abstract}
In this paper, we consider a repair-cost limit replacement problem and develop a graphical method to determine the optimal repair-cost limit which minimizes the expected cost per unit time in the steady-state, using the Lorenz transform of the underlying repair-cost distribution function. The method proposed can be applied to an estimation problem of the optimal repair-cost limit from empirical repair-cost data. Numerical examples are devoted to examine asymptotic properties of the non-parametric estimator for the optimal repair-cost limit.
\end{abstract}

\section{Introduction}

The repair-cost limit replacement policies can provide how to design the recovery mechanism of a system using two maintenance options; repair and replacement, in terms of cost minimization. That is, if the repair cost of a failed unit is greater than the replacement cost, one should replace a failed unit, otherwise one should repair it. First this problem was considered by Drinkwater and Hastings [5] and Hastings [8] for army vehicles. Especially, Hastings [8] proposed three methods of optimizing the repair-cost limit replacement policies by simulation, hill-climbing and dynamic programming. Since the seminal contributions above, a number of authors dealt with a variety of repair-cost limit replacement problems. For instance, Nakagawa and Osaki [15] and Kaio and Osaki [9] reformulated the Hastings' original problem from the viewpoint of renewal reward argument and discussed both continuous and discrete models. Love, Rodger and Blazenko [14] examined the similar problem for vehicle replacement using postal Canada data which was constructed by dividing the life of the vehicle into discrete ages. Park [16] considered a simple but interesting cost limit replacement policy under minimal repair. Love and Guo [13] extended the repair-limit analysis by incorporating a changing force of mortality as the unit ages in the framework of a Markov or semi-Markov decision process.

As Love and Guo [13] pointed out implicitly, it is often assumed that the repair-cost distribution function is arbitrary but known. Of course, this seems to be rather restrictive in many practical situations. In other words, practitioners have to determine the repair-cost limit under incomplete information on the repair-cost distribution in most cases. Dohi, Koshimae, Kaio and Osaki [4] proposed a non-parametric estimator of the optimal repaircost limit from the empirical cost data. More precisely, they applied the total time on test (TTT) statistics to those estimation problems in accordance with the graphical idea by Bergman [1] and Bergman and Klefsjö [2]. If the optimal repair-cost limit has to be estimated from the sample data with unknown repair-cost distribution, their method will be useful in practice, since one need not specify the repair-cost distribution in advance.

However, it should be noted that the repair-cost limit replacement problem in [4] was 
very interesting but somewhat different from existing ones. More specifically, the main objective in [4] was to derive the optimal repair-cost limit to retire the repair action, i.e. if the repair is not completed within a cost limit, the failed unit is scrapped and then a new spare is ordered. Such a policy seems to be plausible in some practical situations, but should be distinguished from the original repair-cost limit problem. In this paper, we consider a repair-cost limit replacement problem proposed by Nakagawa and Osaki [15] in the framework of renewal reward processes and propose a statistical estimation method based on the Lorenz curve. Notice that the basic idea in this paper is similar to the graphical one used in [4] but the statistical device employed here is different from the TTT statistics. The Lorenz curve was first introduced by Lorenz [12] into economics to describe income distributions. Since the Lorenz curve is essentially equivalent to the Pareto curve used in the quality control, it will be one of the most important statistics in every social sciences.

The more general and tractable definition of the Lorenz curve was made by Gastwirth [6]. Goldie [7] proved the strong consistency of the empirical Lorenz curve and discovered its several convergence properties. Chandra and Singpurwalla [3] and Klefsjö [10] investigated the relationship between the TTT statistics and the Lorenz statistics, and derived a few aging and partial ordering properties. Recently, the further results on two statistics were examined by Pham and Turkkan [18] and Perez-Ocon, Gamiz-Perez and Ruiz-Castro [17]. It is shown that the estimator of the optimal repair-cost limit derived in this paper has also several powerful properties proved in earlier contributions above.

The paper is organized as follows. In Section 2, we describe the repair-cost limit replacement problem under consideration. In Section 3 we develop a graphical method to calculate the optimal repair-cost limit which minimizes the expected cost per unit time in the steady-state. Then, it is seen that the Lorenz curve plays an important role to derive the optimal solution on the graph. In Section 4, the statistical estimation problem is discussed. We show that the estimator of the optimal repair-cost limit has a strong consistency, and examine its convergence property. Numerical examples are presented for illustration of the graphical method throughout the paper.

\section{Model Description}

Consider a single-unit repairable system, where each spare is provided only by an order after a lead time $L(>0)$ and each failed unit is repairable. The original unit begins operating at time 0 and the mean time to failure for each unit is $m_{f}(>0)$. When the unit has failed, the decision maker wishes to determine whether he or she should repair it or order a new spare. If the decision maker estimates that the repair is completed within a prespecified cost limit $v_{0} \in[0, \infty)$, then the repair is started immediately at the failure time. The mean repair time is $m_{s}(>0)$ when the repair cost does not exceed $v_{0}$. On the other hand, if the decision maker estimates that the repair cost exceeds the cost limit $v_{0}$, then the failed unit is scrapped immediately and a new spare unit is ordered. Then the spare unit is delivered after the lead time $L$. Without any loss of generality, it is assumed that the unit once repaired is presumed as good as new and that the time required for replacement is negligible.

The repair cost $V$ for each unit is a non-negative i.i.d. random variable and unknown. The decision maker has a subjective probability distribution function $\operatorname{Pr}\{V \leq v\}=H(v)$ on the repair cost, with density $h(v)(>0)$ and finite mean $m_{m}(>0)$. Suppose that the distribution function $H(v) \in[0,1]$ is arbitrary, absolutely continuous and strictly increasing in $v \in[0, \infty)$, and has an inverse function, i.e. $H^{-1}(\cdot)$. Under these model assumptions, define the interval from the start of the operation to the following start as one cycle. Figure 


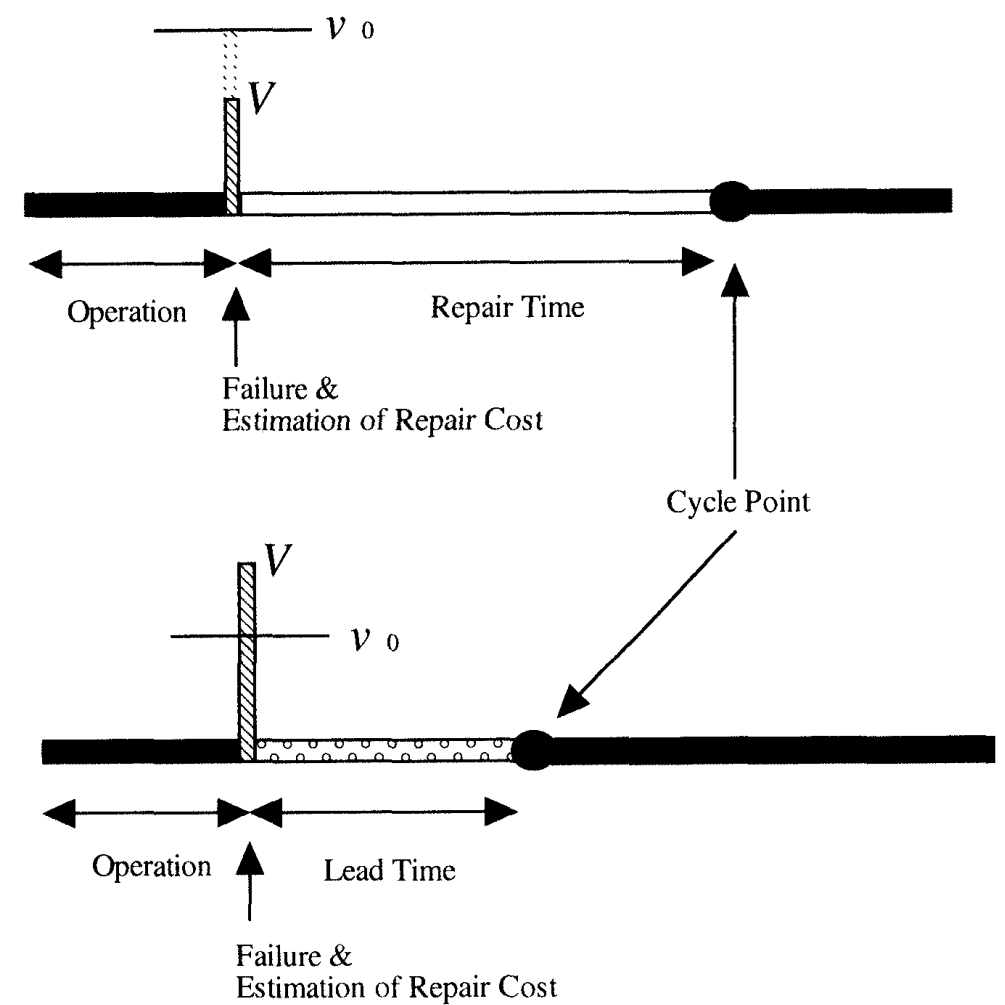

Figure 1: Configuration of repair-cost limit replacement problem.

1 depicts the configuration of the model under consideration. The costs considered in this paper are the following;

$k_{f}(>0)$ : a cost per unit shortage time.

$c(>0)$ : a cost for each order.

We make the following additional assumptions:

(A-1) $m_{s}>L$.

(A-2) $k_{f} m_{s}<k_{f} L+c$.

The assumption (A-1) implies that the mean repair time $m_{s}$ is strictly longer than the lead time. In the assumption (A-2), the shortage cost when the repair cost does not exceed $v_{0}$ is less than the total cost when the new spare is ordered. It is noticed that these assumptions motivate the underlying problem to determine the optimal repair-cost limit.

Let us formulate the expected cost during one cycle. If the decision maker judges that a new spare unit should be ordered, then the ordering cost for one cycle is $c \bar{H}\left(v_{0}\right)$, where $\bar{H}(\cdot)=1-H(\cdot)$. In this case, the expected shortage cost for one cycle is $k_{f} L \bar{H}\left(v_{0}\right)$. On the other hand, if he or she selects the repair option, the expected repair cost for one cycle is $\int_{0}^{v_{0}} v d H(v)$ and the expected shortage cost for one cycle is $k_{f} m_{s} H\left(v_{0}\right)$. Thus the total expected cost during one cycle is

$$
E_{C}\left(v_{0}\right)=\int_{0}^{v_{0}} v d H(v)+k_{f}\left\{m_{s} H\left(v_{0}\right)+L \bar{H}\left(v_{0}\right)\right\}+c \bar{H}\left(v_{0}\right) .
$$

Also, the mean time length of one cycle is

$$
E_{T}\left(v_{0}\right)=m_{f}+m_{s} H\left(v_{0}\right)+L \bar{H}\left(v_{0}\right)
$$


It may be appropriate to adopt an expected cost per unit time in the steady-state over an infinite planning horizon. The expected cost per unit time in the steady-state is, from the renewal reward argument,

$$
T C\left(v_{0}\right)=\lim _{t \rightarrow \infty} \frac{\mathrm{E}[\text { the total cost on }(0, t]]}{t}=E_{C}\left(v_{0}\right) / E_{T}\left(v_{0}\right)
$$

The problem is to derive the optimal repair-cost limit $v_{0}{ }^{*}$ such as

$$
T C\left(v_{0}^{*}\right)=\min _{0 \leq v_{0}<\infty} T C\left(v_{0}\right)
$$

Then, we have the following result on the optimal repair-cost limit.

Theorem 2.1: Define the numerator of the derivative of Eq.(3) with respect to $v_{0}$, divided by $h\left(v_{0}\right)$, as $q_{0}\left(v_{0}\right)$, i.e.

$$
q_{0}\left(v_{0}\right) \equiv\left\{v_{0}+k_{f} m_{s}-k_{f} L-c\right\} E_{T}\left(v_{0}\right)-\left\{m_{s}-L\right\} E_{C}\left(v_{0}\right)
$$

Suppose that both assumptions (A-1) and (A-2) hold. Then there exists a unique optimal repair-cost limit $v_{0}^{*}\left(0<v_{0}^{*}<\infty\right)$ satisfying $q_{0}\left(v_{0}^{*}\right)=0$ and the minimum expected cost is

$$
T C\left(v_{0}^{*}\right)=\frac{v_{0}^{*}+k_{f} m_{s}-k_{f} L-c}{m_{s}-L} .
$$

Proof: The proof is similar to Nakagawa and S. Osaki [15]. Differentiating $T C\left(v_{0}\right)$ with respect to $v_{0}$ and setting equal to zero implies $q_{0}\left(v_{0}\right)=0$. This leads to $d q_{0}\left(v_{0}\right) / d v_{0}=$ $E_{T}\left(v_{0}\right)>0$ and the fact that the function $T C\left(v_{0}\right)$ is strictly convex in $v_{0}$. Since $\lim _{v_{0} \rightarrow \infty} q\left(v_{0}\right)$ $\rightarrow \infty$ and $q(0)<0$ under $(\mathrm{A}-1)$ and $(\mathrm{A}-2)$, there exists a unique optimal repair-cost limit $v_{0}^{*}\left(0<v_{0}^{*}<\infty\right)$ satisfying $q_{0}\left(v_{0}^{*}\right)=0$. The proof is completed.

(Q.E.D.)

From Theorem 2.1, one sees that the optimal repair-cost limit can be calculated easily, by solving the nonlinear equation $q_{0}\left(v_{0}\right)=0$, if the repair-cost distribution is completely known. In the following section, the minimization problem in Eq.(4) is transformed to a simple graphical one on the Lorenz curve.

\section{Graphical Method}

Instead of differentiating $T C\left(v_{0}\right)$ with respect to $v_{0}$ directly, we here employ an interesting graphical method. Define the Lorenz transform of the repair-cost distribution $p \equiv H(v)$ by

$$
\phi(p)=\frac{1}{m_{m}} \int_{0}^{p} H^{-1}(v) d v, \quad 0 \leq p \leq 1
$$

Then the curve $\mathcal{L}=\{(p, \phi(p)) ; p \in[0,1]\}$ is called the Lorenz curve [6, 7]. It should be noted that the curve $\mathcal{L}$ is absolutely continuous from the continuity of $H(v)$. The following result plays an important role to develop the graphical solution method.

Theorem 3.1: Suppose that the assumption (A-1) holds. The minimization problem in Eq.(4) is equivalent to

$$
\min _{0 \leq p \leq 1}: M(p, \phi(p)) \equiv \frac{\phi(p)+\xi}{p+\eta}
$$


where

$$
\xi=\frac{c m_{s}-\left\{k_{f}\left(m_{s}-L\right)-c\right\} m_{f}}{m_{m}\left(m_{s}-L\right)}
$$

and

$$
\eta=\frac{m_{f}+L}{m_{s}-L}
$$

Proof: From the definition of the Lorenz transform in Eq.(7), we have

$$
\begin{aligned}
T C\left(v_{0}\right) & =T C\left(H^{-1}(p)\right) \\
& =\frac{\phi(p)+\left(k_{f} L+c\right) / m_{m}+\left[\left(k_{f}\left(m_{s}-L\right)-c\right] p / m_{m}\right.}{\left(m_{f}+L\right) / m_{m}+\left(m_{s}-L\right) p / m_{m}} \\
& =\left(\frac{m_{m}}{m_{s}-L}\right) \frac{\phi(p)+\xi}{p+\eta}+\frac{k_{f}\left(m_{s}-L\right)-c}{m_{s}-L} .
\end{aligned}
$$

From (A-1) and the continuity of $H(v)$, the optimal $p^{*} \in[0,1]$ which minimizes $T C\left(H^{-1}(p)\right)$ is a solution of the minimization problem in Eq.(8). The proof is thus completed. (Q.E.D.)

From Theorem 3.1, the optimal repair-cost limit is determined by $p^{*}=H\left(v_{0}^{*}\right)$ which minimizes the tangent slope from the point $\mathrm{B}=(-\eta,-\xi) \in(-\infty, 0) \times(-\infty, 0)$ to the curve $\mathcal{L}$ in the plane $(x, y) \in[0,1] \times[0,1]$ under the assumption $(\mathrm{A}-2)$.

More precisely, we prove the uniqueness of the optimal repair-cost limit.

Theorem 3.2: Suppose that both assumptions (A-1) and (A-2) hold. Then there exists a unique optimal solution $p^{*}=H\left(v_{0}^{*}\right)\left(0<v_{0}^{*}<\infty\right)$ minimizing $M(p, \phi(p))$, where $p^{*}$ is given by the $x$-coordinate at the point of contact for the curve $\mathcal{L}$ from the point $\mathrm{B}$.

Proof: From (A-1) and (A-2), it can be seen that $\xi>0$ and $\eta>0$. Differentiating $M(p, \phi(p))$ with respect to $p$ and setting it equal to zero implies

$$
q(p) \equiv(d \phi(p) / d p)(p+\eta)-(\phi(p)+\xi)
$$

where $d \phi(p) / d p=H^{-1}(p) / m_{m}$. Further, we have

$$
d q(p) / d p=d^{2} \phi(p) / d p^{2}(p+\eta)>0
$$

and the function $M(p, \phi(p))$ is strictly convex in $p$, since $d^{2} \phi(p) / d p^{2}=1 /\left\{m_{m} h\left(H^{-1}(p)\right)\right\}>$ 0 . From $q(0)=-\xi<0$ and $q(1) \rightarrow \infty$, the proof is completed.

The result above is a dual theorem and is essentially same as Theorem 2.1. The interesting point of Theorem 3.2 is to determine the optimal solution on the graph instead of solving the nonlinear equation.

Example 3.3: We give an example for the graphical method proposed above. Suppose that the repair-cost distribution $H(v)$ is known and obeys the Weibull distribution;

$$
H(v)=\exp \left\{-\left(\frac{t}{\theta}\right)^{\beta}\right\}
$$

with the shape parameter $\beta=4.0$ and the scale parameter $\theta=0.9$. The other model parameters are $c=0.4000(\$), L=0.3500$ (day), $k_{f}=0.4000$ (\$/day) $m_{f}=0.3000$ (day), 


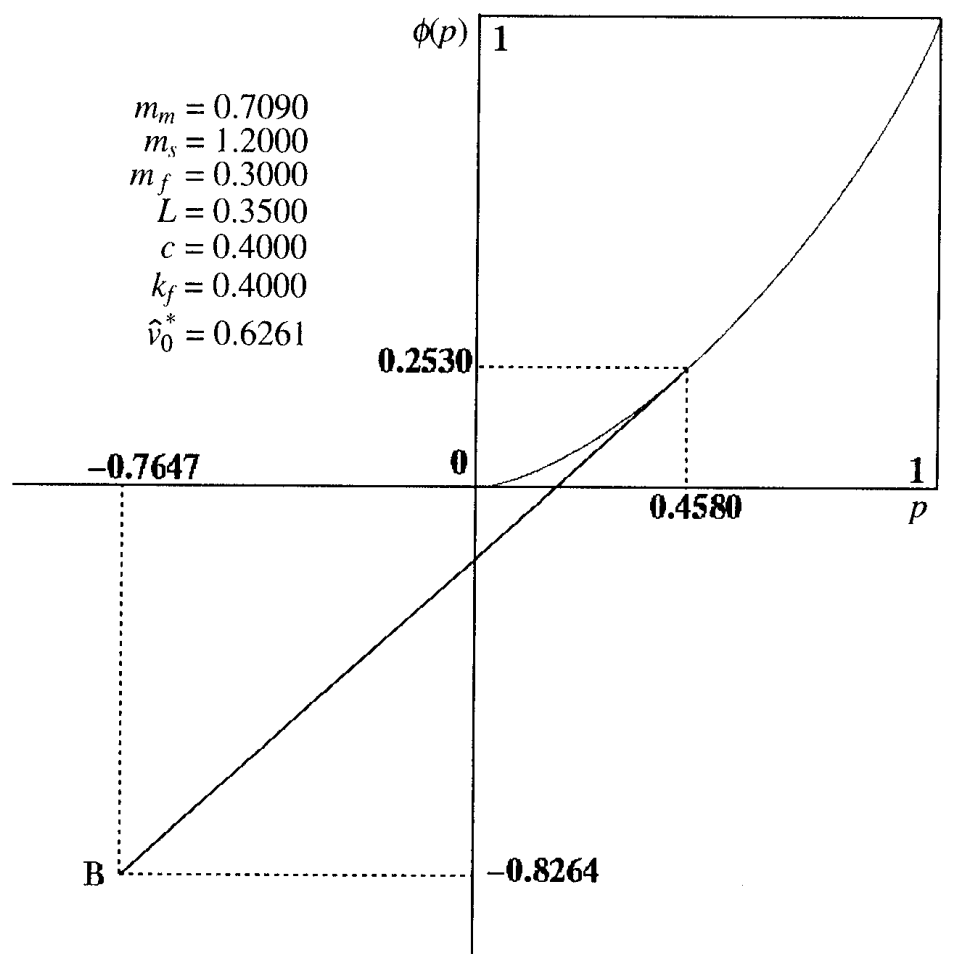

Figure 2: Determination of optimal repair-cost limit on the Lorenz curve.

$m_{s}=1.2000$ (day) and $m_{m}=0.7090(\$)$. The determination of the optimal repair-cost limit is presented in Figure 2. In this case, we have $\mathrm{B}=(-0.7647,-0.8264)$ and the optimal point with minimum slope from $\mathrm{B}$ is $\left(p^{*}, \phi\left(p^{*}\right)\right)=(0.4580,0.2530)$. Thus, the optimal repair-cost limit is $v_{0}^{*}=H^{-1}(0.4580)=0.6261$.

The basic idea for the graphical method proposed in this section can be applied to an estimation problem of the optimal repair-cost limit when the empirical repair-cost data are available. In the following section, the statistical optimization technique is developed for the empirical counterparts of the Lorenz curve.

\section{Statistical Estimation Method}

Based on the graphical idea in Section 3, we propose a non-parametric method to estimate the optimal repair-cost limit. Suppose that the optimal repair-cost limit has to be estimated from an ordered complete sample $0=x_{0} \leq x_{1} \leq x_{2} \leq \cdots \leq x_{n}$ of repair cost data from an absolutely continuous repair-cost distribution $H$, which is unknown. The estimator of $H(v)=p$ is the empirical distribution given by

$$
H_{n}(x)=\left\{\begin{array}{ccc}
i / n & \text { for } & x_{i} \leq x<x_{i+1} \\
1 & \text { for } & x_{n} \leq x
\end{array}\right.
$$

where $i=0,1,2, \cdots, n-1$. Then the Lorenz statistics [7] is defined as

$$
\phi_{n} \equiv\left\{\begin{array}{ccc}
0 & \text { for } & i=0 \\
\sum_{k=1}^{i} x_{k} / \sum_{k=1}^{n} x_{k} & \text { for } & i=1,2, \cdots, n .
\end{array}\right.
$$

Plotting the point $\left(i / n, \sum_{k=1}^{i} x_{k} / \sum_{k=1}^{n} x_{k}\right), i=0,1,2, \cdots, n$, and connecting them by line segments, we obtain the empirical Lorenz curve $\mathcal{L}_{n}=\left\{\left(H_{n}, \phi_{n}\right) ; i=0,1, \cdots, n\right\}$. As em- 


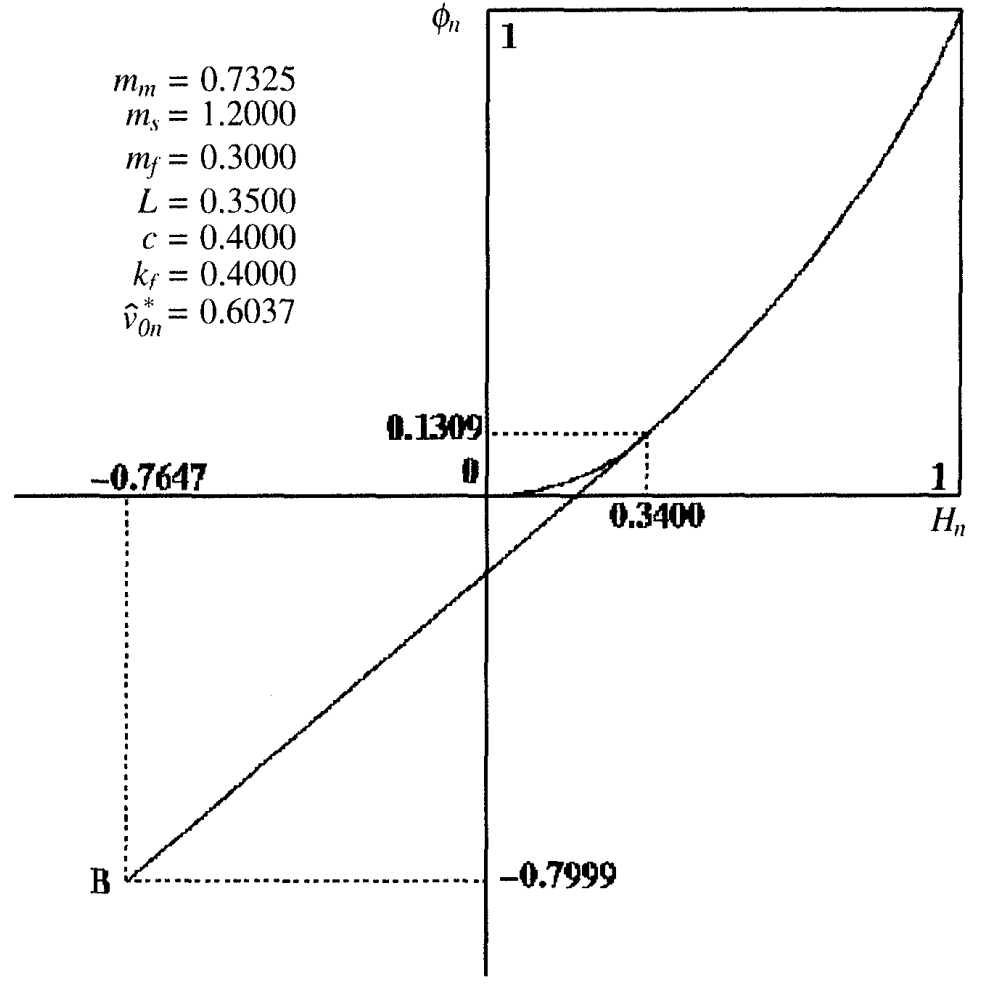

Figure 3: Estimation of optimal repair-cost limit on the empirical Lorenz curve.

pirical counterpart of Theorem 3.1, we obtain a non-parametric estimator of the optimal repair-cost limit in the following theorem.

Theorem 4.1: (i) The optimal repair-cost limit can be estimated by $\hat{v}_{0 n}^{*}=x_{i^{*}}$, where

$$
\left\{i^{*} \mid \min _{0 \leq i \leq n} \frac{\sum_{k=1}^{i} x_{k} / \sum_{k=1}^{n} x_{k}+\xi}{i / n+\eta}\right\}
$$

and the theoretical mean $m_{m}$ involved in $\xi$ is replaced by the sample mean $\sum_{k=1}^{n} x_{k} / n$.

(ii) The estimator $\hat{v}_{0 n}^{*}=x_{i^{*}}$ in Eq.(17) is strongly consistent, i.e. $\hat{v}_{0 n}^{*}=x_{i^{*}} \rightarrow v_{0}^{*}$ as $n \rightarrow \infty$.

The result in (i) is trivial. The proof of (ii) is based on the asymptotic property $\phi_{n} \rightarrow \phi(p)$ as $n \rightarrow \infty$, which is due to Goldie [7].

Example 4.2: The repair-cost data were made by the random number following the Weibull distribution with shape parameter $\beta=4.0$ and scale parameter $\theta=0.9$. The other model parameters are same as Example 3.3 except that $m_{m}$ and $\xi$. The empirical Lorenz curve based on the 30 sample data is shown in Figure 3 , where $m_{m}=\sum_{k=1}^{30} x_{k} / 30=0.7325$ $(\$)$. Since $\mathrm{B}=(-0.7647,-0.7999)$, the optimal point with minimum slope from $\mathrm{B}$ becomes $\left(i^{*} / n, \sum_{k=1}^{i^{*}} x_{k} / \sum_{k=1}^{n} x_{k}\right)=\left(14 / 30, \sum_{k=1}^{14} x_{k} / \sum_{k=1}^{30} x_{k}\right)=(0.3400,0.1309)$. Thus, the estimator of the optimal repair-cost limit is $\hat{v}_{0 n}^{*}=0.6037(\$)$.

If the estimator $\hat{v}_{0 n}^{*}=x_{i^{*}}$ is obtained, it is easy to calculate the estimate of the minimum expected cost. That is, from Eq.(6),

$$
T C\left(\hat{v}_{0 n}^{*}\right)=\frac{\hat{v}_{0 n}^{*}+k_{s} m_{s}-k_{f} L-c}{m_{s}-L},
$$




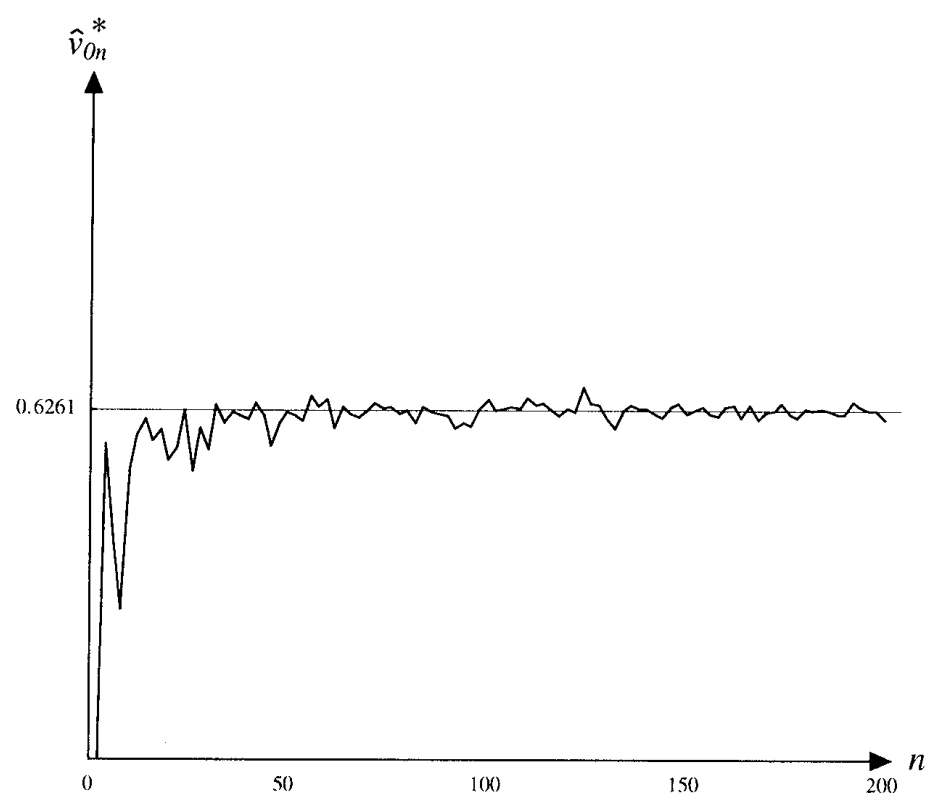

Figure 4: Asymptotic property of estimates for the optimal repair-cost limit.

which may be strongly consistent.

Of our next interest is the convergence speed of the estimators $\hat{v}_{0 n}^{*}$ and $T C\left(\hat{v}_{0 n}^{*}\right)$. We examine numerically the strong consistency of the estimator derived in Theorem 4.1.

Example 4.3: Suppose that the repair-cost distribution and model parameters are similar to those in Example 4.2. Notice that the mean repair cost $m_{m}$ and its associated oarameter $\xi$ are changed in each sample. Then the real optimal repair-cost limit and the minimum expected cost become $v_{0}^{*}=0.6261(\$)$ and $T C\left(v_{0}^{*}\right)=0.6666(\$)$, respectively. On the other hand, the asymptotic behaviours of estimates for the optimal repair-cost limit and their associated minimum expected cost are depicted in Figures 4 and 5, respectively. From these figures, we observe that the estimates converge to the corresponding real values around where the number of data is 30 . In other words, without specifying the repair-cost distribution, the proposed non-parametric method may function to estimate the optimal repair-cost limit precisely.

Finally, utilizing the results above, we determine the asymptotic valid confidence interval for the optimal repair-cost limit approximately. Recall that determining asymptotically valid confidence intervals for the probability distribution function is based on the normal approximation to the binomial distribution. Notice that the empirical distribution defined in Eq.(14) can be regarded as the binomial random variable having mean $\mathrm{E}\left[H_{n}(x)\right]=H(x)$ and variance $\operatorname{Var}\left[H_{n}(x)\right]=H(x) \bar{H}(x) / n$. Furthermore, when the sample size $n$ is large and $H(x)$ is not too close to 0 or 1 , the binomial distribution may have a shape that is closely approximated by a normal distribution, and can be used to find interval estimates for $H(x)$. It should be noted that these interval estimates are most accurate around the median of the distribution, since the normal approximation to the binomial distribution works best when the probability of success is about 0.5 , where the binomial distribution is symmetric. 


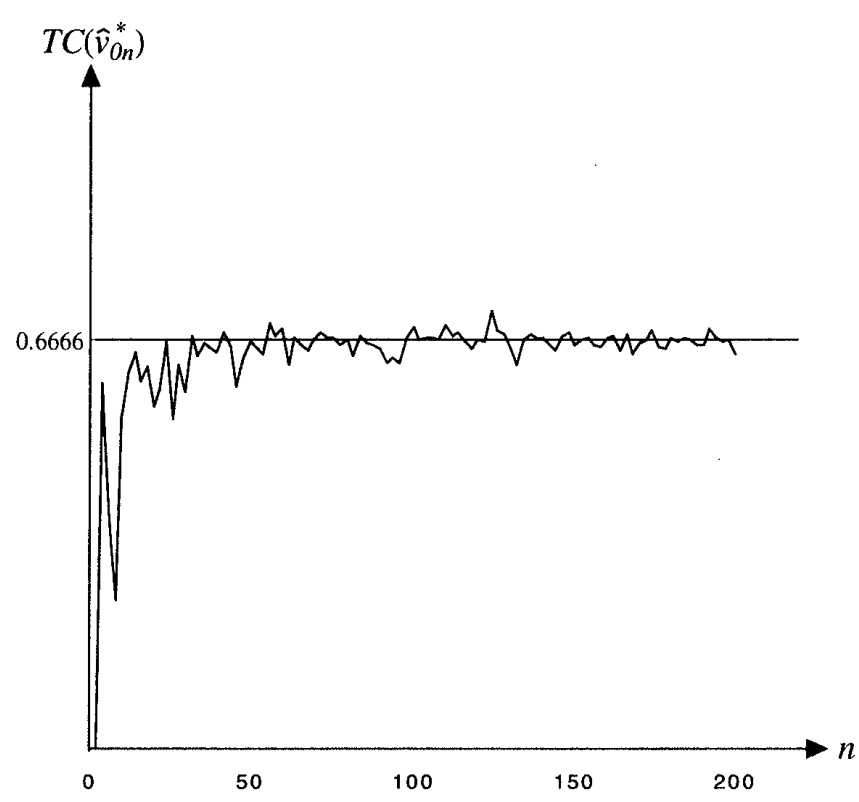

Figure 5: Asymptotic property of estimates for the associated minimum expected cost.

Replacing $H(v)$ by $H_{n}$ in the variance formula, an asymptotically valid $100(1-\alpha) \%$ confidence interval for the repair-cost distribition is approximately

$$
H_{n}^{L}<H(v)<H_{n}^{U}
$$

where $z_{\alpha / 2}$ is the $(1-\alpha / 2)$ fractile of the standard normal distribution, and the lower and upper bounds are

$$
H_{n}^{L}=H_{n}-z_{\alpha / 2} \sqrt{\frac{H_{n} \bar{H}_{n}}{n}}
$$

and

$$
H_{n}^{U}=H_{n}+z_{\alpha / 2} \sqrt{\frac{H_{n} \bar{H}_{n}}{n}}
$$

respectively. This confidence interval is appropriate as the number of data increases (see Lee [11]). Consider the asymptotically valid $100(1-\alpha) \%$ confidence interval for the repaircost distribition. Since the Lorenz curve is a non-decreasing function, the lower and upper bounds of the Lorenz transform are approximately given by

$$
\phi_{n}^{L}<\phi\left(H^{-1}(v)\right)<\phi_{n}^{U}
$$

where

$$
\phi_{n}^{L}=\sum_{k=1}^{\left[n H_{n}^{L}\right]} x_{k} / \sum_{k=1}^{n} x_{k}
$$




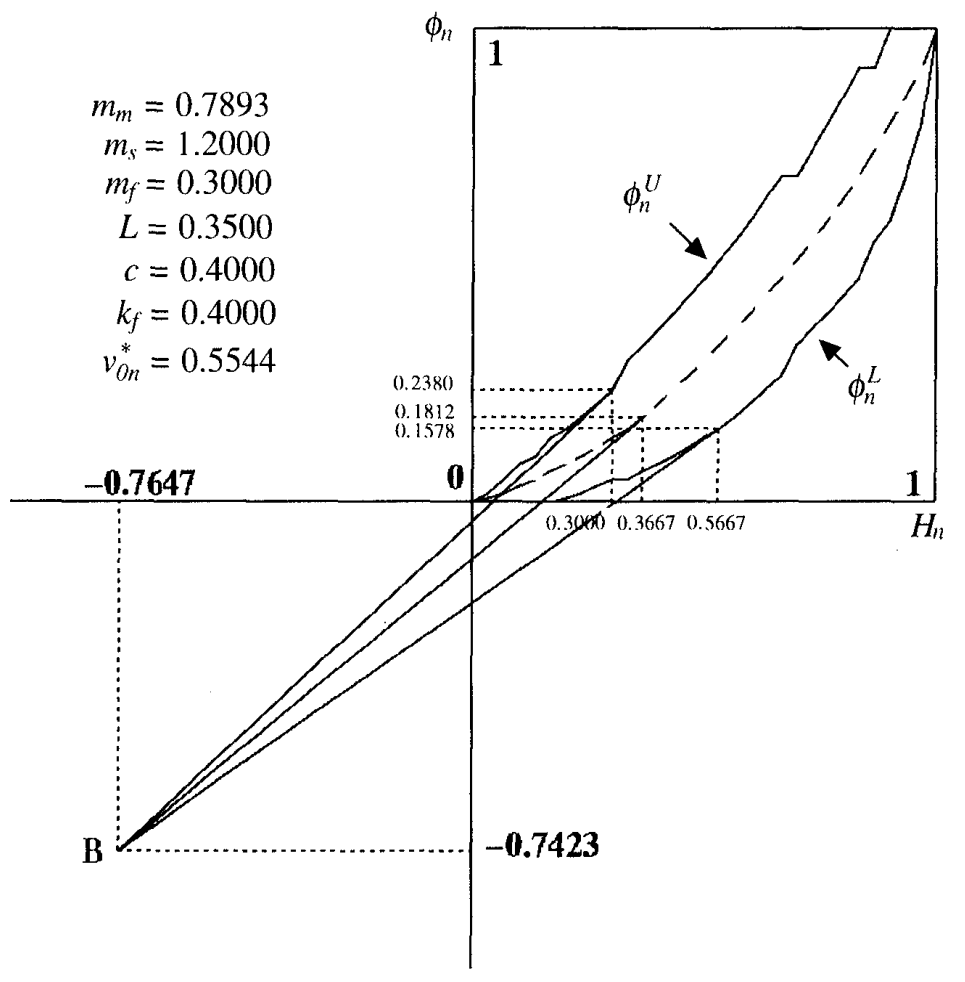

Figure 6: Upper and lower bounds of the empirical Lorenz curve.

$$
\phi_{n}^{U}=\sum_{k=1}^{\left[n H_{n}^{U}\right]} x_{k} / \sum_{k=1}^{n} x_{k}
$$

and $[a]$ is the greatest integer in $a$. Then, it is straightforward to see that the asymptotically valid $100(1-\alpha) \%$ confidence interval for the optimal repair-cost limit is approximately given by

$$
v_{0 n}^{L}<\hat{v}_{0 n}^{*}<v_{0 n}^{U}
$$

where $v_{0 n}^{U}=x_{j^{*}}$ and $v_{0 n}^{L}=x_{k^{*}}$ satisfy

$$
\left\{j^{*} \mid \min _{0 \leq j \leq n} \frac{\sum_{k=1}^{\left[n H_{n}^{L}\right]} x_{k} / \sum_{k=1}^{n} x_{k}+\xi}{j / n-z_{\alpha / 2} \sqrt{(j / n)(1-j / n) / n}+\eta}\right\}
$$

and

$$
\left\{k^{*} \mid \min _{0 \leq k \leq n} \frac{\sum_{k=1}^{\left[n H_{n}^{U}\right]} x_{k} / \sum_{k=1}^{n} x_{k}+\xi}{j / n+z_{\alpha / 2} \sqrt{(j / n)(1-j / n) / n}+\eta}\right\}
$$

respectively.

Example 4.5: Under the same parameters as Example 4.3, we determine the asymptotic valid confidence interval for the optimal repair-cost limit. Figure 6 shows the upper and lower bounds of the empirical Lorenz curve. From this figure, we obtain $v_{0 n}^{L}$ and $v_{0 n}^{U}$ which minimize the tangent slope from the point B to the curves $\phi_{n}^{U}$ and $\phi_{n}^{L}$, respectively. Figures 
7 and 8 show the behaviours of the asymptotically valid $95 \%$ confidence intervals of the optimal repair-cost limit and its associated minimum expected cost, respectively. These figures tell us that the estimation when the number of data is more than 30 is stable, and that the observation result in Example 4.3 can be also valid taking account of the asymptotically confidence interval.

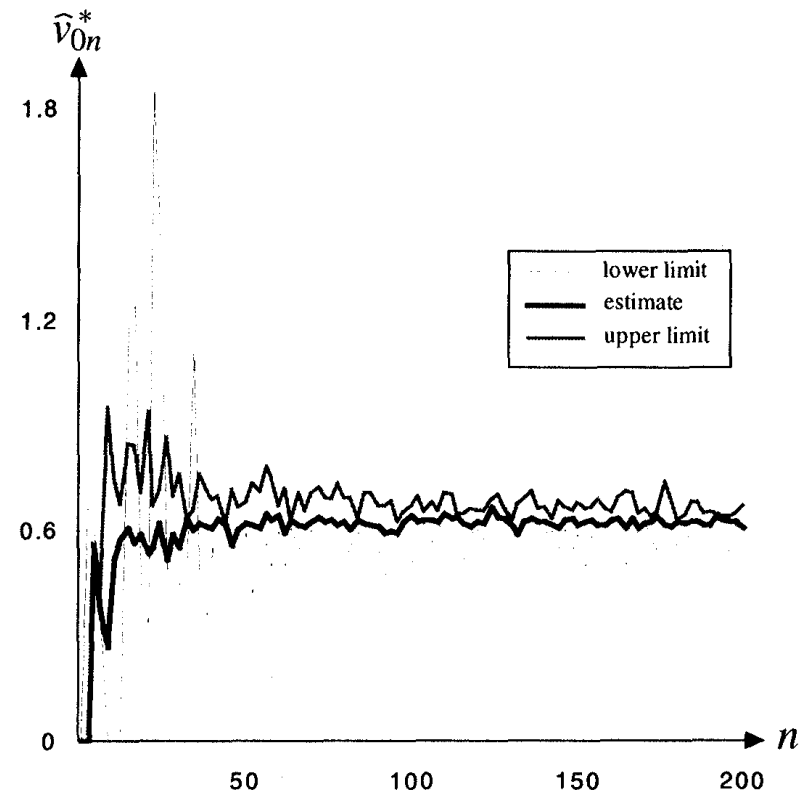

Figure 7: The asymptotically valid $100(1-\alpha) \%$ confidence interval of the optimal repair-cost limit.

\section{Conclusion}

This paper has considered a typical repair-cost limit replacement problem and developed a graphical method to determine the optimal repair-cost limit which minimizes the expected cost per unit time in the steady-state, using the Lorenz transform of the underlying repaircost distribution function. We have examined some properties of the strongly consistent estimator and the asymptotically valid $100(1-\alpha) \%$ confidence interval for the optimal repair-cost limit throughout numerical examples.

The main contribution of this paper is to show that the Lorenz statistics as well as TTT statistics is a useful device to estimate the optimal maintenance schedule. This simple but interesting idea should be applied to solve other kinds of stochastic maintenance optimization problems in the future.

\section{Acknowledgement}

The present research was supported by a Grant-in-Aid for Scientific Research from the Ministry of Education, Sports, Science and Culture of Japan under Grant No. 13780367 and the Research Program under the Institute for Advanced Studies of the Hiroshima Shudo University, Hiroshima 731-3151, Japan. The authors are very grateful to Farid S. Othman, Faculty 


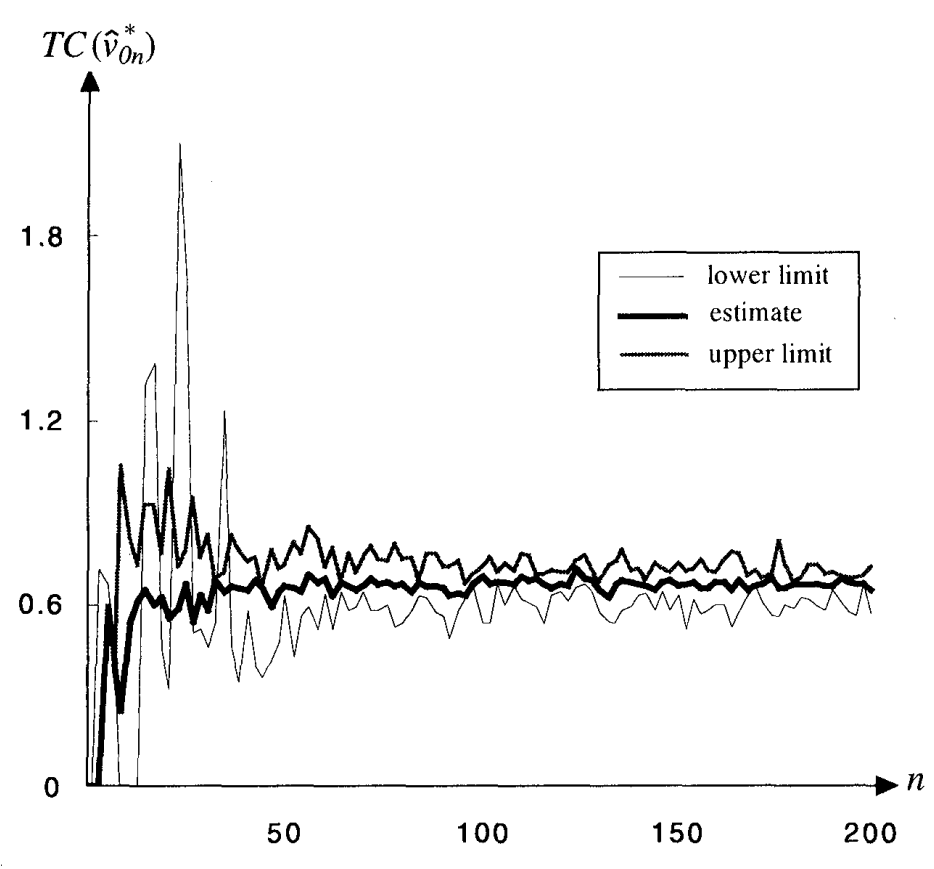

Figure 8: The asymptotically valid $100(1-\alpha) \%$ confidence interval of the minimum expected cost.

of Engineering, Hiroshima University, for his numerical assistance, and to the referees for their useful comments.

\section{References}

[1] B. Bergman: On age replacement and the total time on test concept. Scandinavian Journal of Statistics, 6 (1979) 161-168.

[2] B. Bergman and B. Klefsjö: The total time on test concept and its use in reliability theory. Operations Research, 32 (1984) 596-607.

[3] M. Chandra and N. D. Singpurwalla: Relationship between some notions which are common to reliability and economics. Mathematics of Operations Research, 6 (1981) 113-121.

[4] T. Dohi, H. Koshimae, N. Kaio and S. Osaki: Geometrical interpretations of repaircost limit replacement policies. International Journal of Reliability, Quality and Safety Engineering, 4 (1997) 309-333.

[5] R. W. Drinkwater and N. A. J. Hastings: An economical replacement model. Operational Research Quarterly, 18 (1967) 121-138.

[6] J. L. Gastwirth: A general definition of the Lorenz curve. Econometrica, 39 (1971) 1037-1039.

[7] C. M. Goldie: Convergence theorems for empirical Lorenz curves and their inverses. Advances in Applied Probability, 9 (1977) 765-791.

[8] N. A. J. Hastings: The repair limit replacement method. Operational Research Quarterly, 20 (1969) 337-349. 
[9] N. Kaio and S. Osaki: Optimum repair limit policies with cost constraint. Microelectronics and Reliability, 21 (1981) 597-599.

[10] B. Klefsjö: Reliability interpretations of some concepts from economics. Naval Research Logistics Quarterly, 31 (1984) 301-308.

[11] L. M. Lee: Reliability: Probabilistic Models and Statistical Methods (Prentice-Hall, New Jersey, 1995).

[12] M. O. Lorenz: Methods of measuring the concentration of wealth. Journal of American Statistical Association, 9 (1893) 209-219.

[13] C. E. Love and R. Guo: Utilizing Weibull failure rates in repair limit analysis for equipment replacement/preventive maintenance decisions. Journal of Operational Research Society, 47 (1996) 1366-1376.

[14] C. E. Love, R. Rodger and G. Blazenko: Repair limit policies for vehicle replacement. INFOR, 20 (1982) 226-237.

[15] T. Nakagawa and S. Osaki: Optimum ordering policies with lead time for an operating unit. R. A. I. R. O. Recherche opérationnelle/Operations Research, 12 (1978) 383-393.

[16] K. S. Park: Cost limit replacement policy under minimal repair. Microelectronics and Reliability, 23 (1983) 347-349.

[17] R. Perez-Ocon, M. L. Gamiz-Perez and J. E. Ruiz-Castro: A study of different aging classes via total time on test transform and Lorenz curves. Applied Stochastic Models and Data Analysis, 13 (1998) 241-248.

[18] T. G. Pham and N. Turkkan: The Lorenz and the scaled total-time-on-test transform curves: a unified approach. IEEE Transactions on Reliability, R-43 (1994) 76-84.

Tadashi Dohi

Department of Information Engineering

Hiroshima University

4-1 Kagamiyama 1 Chome

Higashi-Hiroshima 739-8527, JAPAN

E-mail: dohi@gal.sys.hiroshima-u.ac.jp 